Fuel re-absorption by thermally treated co-deposited carbon layers

This article has been downloaded from IOPscience. Please scroll down to see the full text article.

2011 Phys. Scr. 2011014006

(http://iopscience.iop.org/1402-4896/2011/T145/014006)

View the table of contents for this issue, or go to the journal homepage for more

Download details:

IP Address: 130.237.45.102

The article was downloaded on 27/01/2012 at 10:15

Please note that terms and conditions apply. 


\title{
Fuel re-absorption by thermally treated co-deposited carbon layers
}

\author{
D Ivanova ${ }^{1}$, M Rubel $^{1}$, V Philipps ${ }^{2}$, B Schweer $^{2}$, P Petersson $^{3}$, \\ M Freisinger ${ }^{2}$ and A Schmidt ${ }^{2}$ \\ ${ }^{1}$ Alfvén Laboratory, Royal Institute of Technology, Association EURATOM-VR, Stockholm, Sweden \\ ${ }^{2}$ Institute for Energy and Climate Research, Plasma Physics, Forschungszentrum Jülich, \\ Association EURATOM-FZJ, Jülich, Germany \\ 3 Ångström Laboratory, Uppsala University, Association EURATOM-VR, Uppsala, Sweden \\ E-mail: Darya.Ivanova@ee.kth.se
}

Received 10 May 2011

Accepted for publication 25 July 2011

Published 16 December 2011

Online at stacks.iop.org/PhysScr/T145/014006

\begin{abstract}
Systematic studies have been conducted to address the fuel re-absorption by carbon deposits under repeated exposure to plasma after cleaning procedures. The investigation was done with graphite tiles from ALT-II (Advanced Limiter Test II), i.e. the main limiter at the TEXTOR tokamak. Pure graphite plates were used as the reference material. The experimental programme comprised the following: pre-characterization of specimens; D desorption by baking the tile at $1273 \mathrm{~K}$; surface analyses of the fuel-depleted layers; exposure to deuterium in a laboratory plasma device and in TEXTOR; and quantitative assessment of deuterium re-absorption. The main result is that fuel retention in the re-exposed deposits is $30-40$ times lower than that in the original co-deposit, showing that fuel re-absorption does not lead to an immediate re-saturation of deposits. Annealing at high temperatures enhances layer brittleness, leading eventually to detachment of co-deposits.
\end{abstract}

PACS number: 52.40.Hf

(Some figures may appear in colour only in the online journal)

\section{Introduction}

Reduction of in-vessel fuel inventory in fusion devices is essential when operation involves a deuterium-tritium plasma [1]. This issue becomes even more important if carbon plasma-facing components (PFCs) are used [2-4]. Two basic schemes for fuel removal are currently considered: (i) desorption of hydrogen-containing species and (ii) removal of the entire fuel-rich co-deposit. The first approach is based on surface heating by photonic means [5-10], ion cyclotron wall conditioning [11] or long-term annealing of PFCs [12]. In all cases, the fuel-depleted layers will remain in the vessel and they would be repeatedly exposed to plasma. The question is: how do such layers respond to plasma during the repeated exposure? Systematic studies have been undertaken to address fuel re-absorption by carbon deposits exposed to plasma after cleaning procedures. The aim was to explore the morphology and behaviour of fuel-depleted surfaces in a deuterium environment, i.e. to assess whether the layers are instantly re-saturated by fuel species and whether the fuel uptake characteristics differ from those observed on fresh carbon targets.

\section{Experimental details}

The investigation was done with deposit-covered specimens obtained by sectioning graphite tiles from the main toroidal pump limiter ALT-II (Advanced Limiter Test II) at the TEXTOR tokamak. Pure graphite plates (EK 98) were used as the reference material. Pre-characterization and post-mortem analyses of all samples were performed by means of gas-phase and material research methods: thermal desorption spectrometry (TDS), ion beam techniques (e.g. nuclear reaction analysis (NRA)) and scanning electron microscopy (SEM).

The ALT-II limiter surface features a pronounced distinction between the erosion and the deposition areas $[13,14]$. The deposition zone on that limiter is also the main 

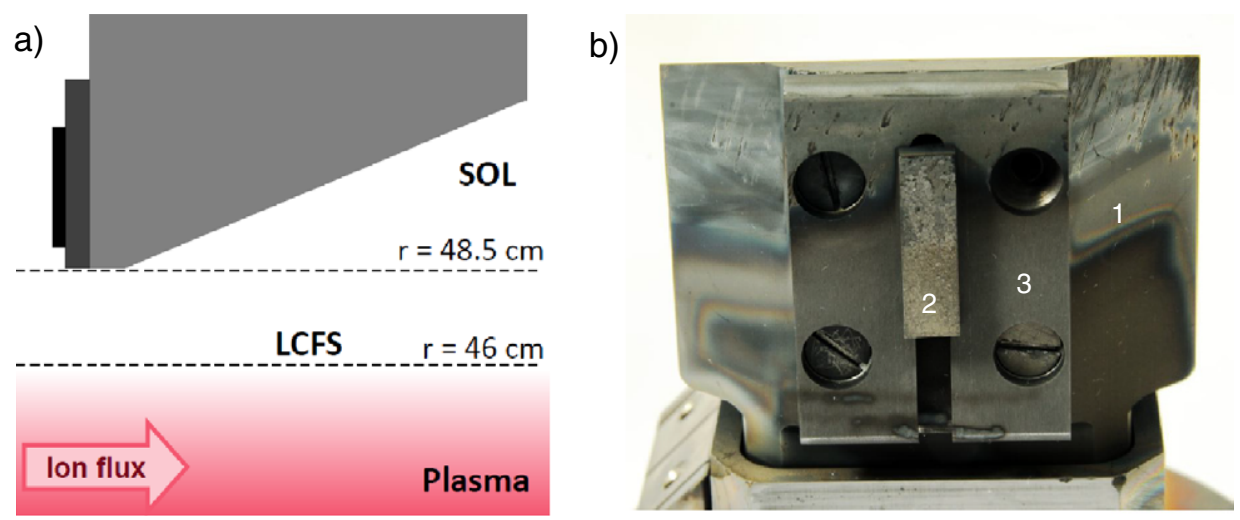

Figure 1. Experimental setup at TEXTOR. (a) Schematic positioning of the single-roof test limiter in relation to plasma; (b) a photograph of a single-roof test limiter after exposure in TEXTOR (1) with a specimen of the ALT-II tile (2) and a pure graphite holder also serving the reference material (3).

area of fuel accumulation in TEXTOR. Therefore, an ALT tile retrieved during the shutdown in 2008 after a long-term campaign was selected for fuel retention and re-absorption studies. A number of specimens were cut from the deposition zone of this tile: the initial surface area of each sample was $2 \mathrm{~cm} \times 3 \mathrm{~cm}$, but the later steps in the experimental programme required further reduction of the specimen size to $1 \mathrm{~cm} \times 3 \mathrm{~cm}$. They were pre-characterized by SEM and NRA and then outgassed at $1273 \mathrm{~K}$ for further experiments. Outgassing of each sample was performed in a TDS chamber with synchronous monitoring of masses $\mathrm{M} 2\left(\mathrm{H}_{2}\right), \mathrm{M} 3$ (HD), M4 ( $\left.\mathrm{D}_{2}\right)$ and masses M19-M20 corresponding to D-containing water and $\mathrm{C}_{1}$ hydrocarbons. In order to avoid oversaturation of the quadrupole due to a massive gas release, a relatively low heating rate was chosen: $0.055 \mathrm{~K} \mathrm{~s}^{-1}$ with the total time required to reach $1273 \mathrm{~K}$ being about $5 \mathrm{~h}$. The same TDS apparatus and desorption parameters were used for the total fuel retention studies of the repeatedly exposed specimens. Exposures to deuterium were performed in laboratory plasma facilities and in TEXTOR.

A low-temperature plasma device, PADOS, operated at the Forschungszentrum Jülich was employed to study fuel re-absorption under laboratory conditions. The chamber is routinely used in the deposition of amorphous deuterated carbon (a-C:H) and boron layers [15]. A glow discharge in the vacuum chamber of PADOS is generated between two circular electrodes. The outgassed limiter plate with a fuel-free deposit and a reference plate were placed on the lower electrode (cathode). The temperature of the cathode was kept at $450 \mathrm{~K}$. Exposure to the glow discharge deuterium plasma was performed for $3 \mathrm{~h}$ with a flux of about $1 \times 10^{15} \mathrm{~cm}^{2} \mathrm{~s}^{-1}$.

The second series of exposures of the outgassed deposits was performed in their native environment, i.e. in the TEXTOR tokamak where these layers were originally formed. The plates for exposure were mounted on a holder that was attached vertically to the side of the test limiter (shaped as a single-roof block) to face the ion flux. The holder for fixing the deposit-containing sample to the roof-shaped block was made of a pure graphite plate, which then served as a reference surface in the retention studies. A scheme of the entire setup is shown in figure 1(a). A photograph taken after exposure (figure 1(b)) demonstrates the frontal view of the test limiter with the attached samples. It was inserted into the vacuum vessel from the top using a transfer system at limiter lock number 3 , which is dedicated to material testing in TEXTOR [16]. The block was positioned in the scrape-off layer (SOL) plasma. The top was at the radial position $r=$ $48.5 \mathrm{~cm}$, i.e. $2.5 \mathrm{~cm}$ behind the last closed flux surface. ALT-II was the main limiter defining the plasma radius at $a=46 \mathrm{~cm}$.

Two exposures were performed as a parasitic experiment during the commissioning of a charge exchange recombination spectrometer. Thus, the experimental programme ensured a variety of discharges, both ohmic and auxiliary, heated by the two neutral beam injectors. The main plasma parameters varied slightly for each discharge in the following ranges: the toroidal magnetic field strengths $B_{\mathrm{t}}=2.2-2.6 \mathrm{~T}$, line averaged electron density $n_{\mathrm{e}}=2.5-3 \times 10^{19} \mathrm{~m}^{-3}$ and plasma current $I_{\mathrm{p}}=350-400 \mathrm{kA}$. For the first experimental campaign, the total exposure time consisted of eight discharges (approximately 40 plasma seconds). During the cleaning glow discharges, the test limiter was retracted beyond the liner position $(r=55 \mathrm{~cm})$. These precautions could not be taken for the second experiment, when the test limiter remained at the same position $(r=48.5 \mathrm{~cm})$ during all conditioning and disruption events, which also resulted in damage to the surface of the test limiter and exposed samples (see figure 1). The total time of steady plasma operation in this case was only $25 \mathrm{~s}$.

The deuterium content on surfaces was measured at all stages. It was determined by NRA using a $2 \mathrm{MeV}{ }^{3} \mathrm{He}^{+}$ beam and by detecting high-energy protons emerging from the reaction $\mathrm{D}\left({ }^{3} \mathrm{He}, \mathrm{p}\right)^{4} \mathrm{He}$. The information depth with the $2 \mathrm{MeV}^{3} \mathrm{He}^{+}$beam in graphite is $6 \mu \mathrm{m}\left(\rho=1.9 \mathrm{~g} \mathrm{~cm}^{-3}\right)$ and $7 \mu \mathrm{m}$ in the deposit ( $\rho$ was assumed to be $1.6 \mathrm{~g} \mathrm{~cm}^{-3}$ ). The depth profiles of the fuel distribution were determined. Comparison of the TDS and NRA measurements of the $\mathrm{D}$ content in the original co-deposits and after repeated exposures has shown that there is good agreement between these methods (within 10\%).

\section{Results and discussion}

The initial fuel content in thick co-deposited layers on the ALT-II tiles is in the range $7-11 \%$, i.e. the 

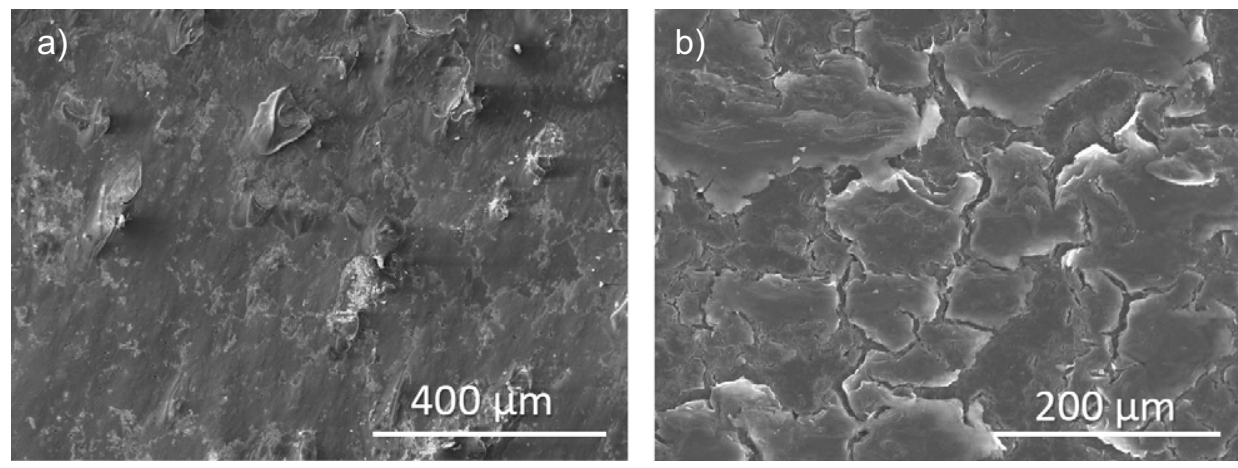

Figure 2. Morphology of the ALT-II surface with deposit: (a) before and (b) after annealing at $1273 \mathrm{~K}$.

deuterium-to-carbon concentration ratio $(\mathrm{D} / \mathrm{C})$ is about 0.1 , as studied in detail after several long-term campaigns in TEXTOR [13, 17, 18]. The micrograph in figure 2(a) demonstrates an SEM image of the limiter surface with deposits (as retrieved from TEXTOR), whereas the limiter surface after annealing at up to $1273 \mathrm{~K}$ is shown in figure 2(b). Thermal treatment causes a strong modification of the deposit structure. It enhances the surface roughness and layer brittleness, eventually leading to flaking and detachment of co-deposits, i.e. dust formation. These effects additionally complicate the subsequent analysis and handling of the deposit-containing samples.

To find possible differences in the fuel content between the original co-deposits and layers exposed to deuterium after outgassing, the following features were measured: (a) surface content of deuterium and depth distribution up to the accessible information depth with NRA and (b) total D amount and desorption characteristic with TDS.

\subsection{Exposure in PADOS}

The original co-deposits on ALT-II, as determined with NRA, contain about $4.7 \times 10^{18} \mathrm{D} \mathrm{cm}^{-2}$ in a layer of up to $7 \mu \mathrm{m}$, i.e. within the accessible depth with a $2 \mathrm{MeV}$ ${ }^{3} \mathrm{He}^{+}$. The outgassed specimen from ALT-II and a graphite reference plate were exposed in PADOS to a glow discharge in deuterium. The resulting depth integrated retention in pure graphite is horizontally uniform at the level of $1.33 \times$ $10^{17} \mathrm{D} \mathrm{cm}^{-2}$. About $70 \%$ of the $\mathrm{D}$ atoms are stored in a thin surface layer $(<1.5 \mu \mathrm{m})$, whereas the rest are detected deeper (up to $4 \mu \mathrm{m}$ ). This depth is larger than what could be explained only by direct implantation of $\mathrm{D}$ atoms. The factors possibly influencing the observed depth distribution are surface imperfections, the formation of a thin deuterated carbon film on the surface and the diffusion of D atoms along the grain boundaries. Fuel retention in the outgassed layer from ALT-II is close to that in the pure graphite sample: $1.44 \times 10^{17} \mathrm{D} \mathrm{cm}^{-2}$. The formation of a thin deuterated film on the surface, as well as on the reference graphite specimen exposed under the same conditions, is noted. However, most of the retained deuterium atoms are distributed evenly in the entire analysed depth. This indicates that re-absorption of deuterium atoms plays some role in the fuel retention in the outgassed and re-exposed deposits, but the concentration is significantly smaller (at least one order of magnitude) when compared with the original amount.
Plots in figures 3(a) and (b) show thermal desorption spectra for deposits from ALT-II; for more details of such deposits, see [18]. The release of species with mass 3, 4, 19 and 20 is plotted: HD (M3), $\mathrm{D}_{2}$ (M4), M19 and M20, which represent a mixture of $\mathrm{C}_{1}$ hydrocarbons and deuterated water (HDO, $\mathrm{D}_{2} \mathrm{O}$ ). The most important feature is that the release occurs predominantly in the temperature range 700-900 K with a peak value around $750 \mathrm{~K}$.

After exposure of the outgassed deposit and a pure graphite plate to deuterium plasma in PADOS, both specimens were heated again to $1273 \mathrm{~K}$ at a steady heating rate of $0.055 \mathrm{~K} \mathrm{~s}^{-1}$. Plots in figure $3(\mathrm{c})$ are the desorption curves of species M19 and M20 from the re-exposed deposit and the reference graphite plate. There are significant differences between the desorption characteristics from the two specimens. For the re-exposed deposit the most significant release occurs at the initial stage of outgassing at temperatures below $600 \mathrm{~K}$. This can most probably be attributed to the presence of water $\left(\mathrm{H}_{2} \mathrm{O}\right)$, which has a similar desorption characteristic. Water vapour is easily adsorbed by porous deposits when a specimen is stored in air. The occurrence of water species of mass 19 and 20 is attributed to isotope exchange leading to the formation of HDO and $\mathrm{D}_{2} \mathrm{O}$ molecules. The value of M19 is approximately two orders of magnitude larger than $\mathrm{M} 20$, because the probability of $\mathrm{D}_{2} \mathrm{O}$ formation is much lower than HDO. This low-temperature release is not detected for the reference graphite sample (no porous structure), where temperatures above $600 \mathrm{~K}$ are needed in order to remove hydrocarbons $\left(\mathrm{CHD}_{3}\right.$ and $\left.\mathrm{CD}_{4}\right)$ efficiently. Desorption curves for M3 and M4 exhibit distinct peaks around 500,750 and $1150 \mathrm{~K}$ for both the re-exposed deposit and the reference graphite (figure 3(d)). The contrast with figure 3(a) can probably be attributed to differences between exposure conditions in the laboratory plasma and in the tokamak which lead to the formation of different binding states.

\subsection{Exposure in TEXTOR}

Two exposures of the outgassed deposits were performed in the SOL plasma of TEXTOR. The results of the exposure in more favourable conditions (i.e. $40 \mathrm{~s}$ of steady plasma with no disruptions and/or cleaning discharges) are summarized in the following. The deuterium radial distribution and content determined by NRA in the re-exposed ALT-II deposit and in the graphite holder (acting as a reference plate) are plotted 

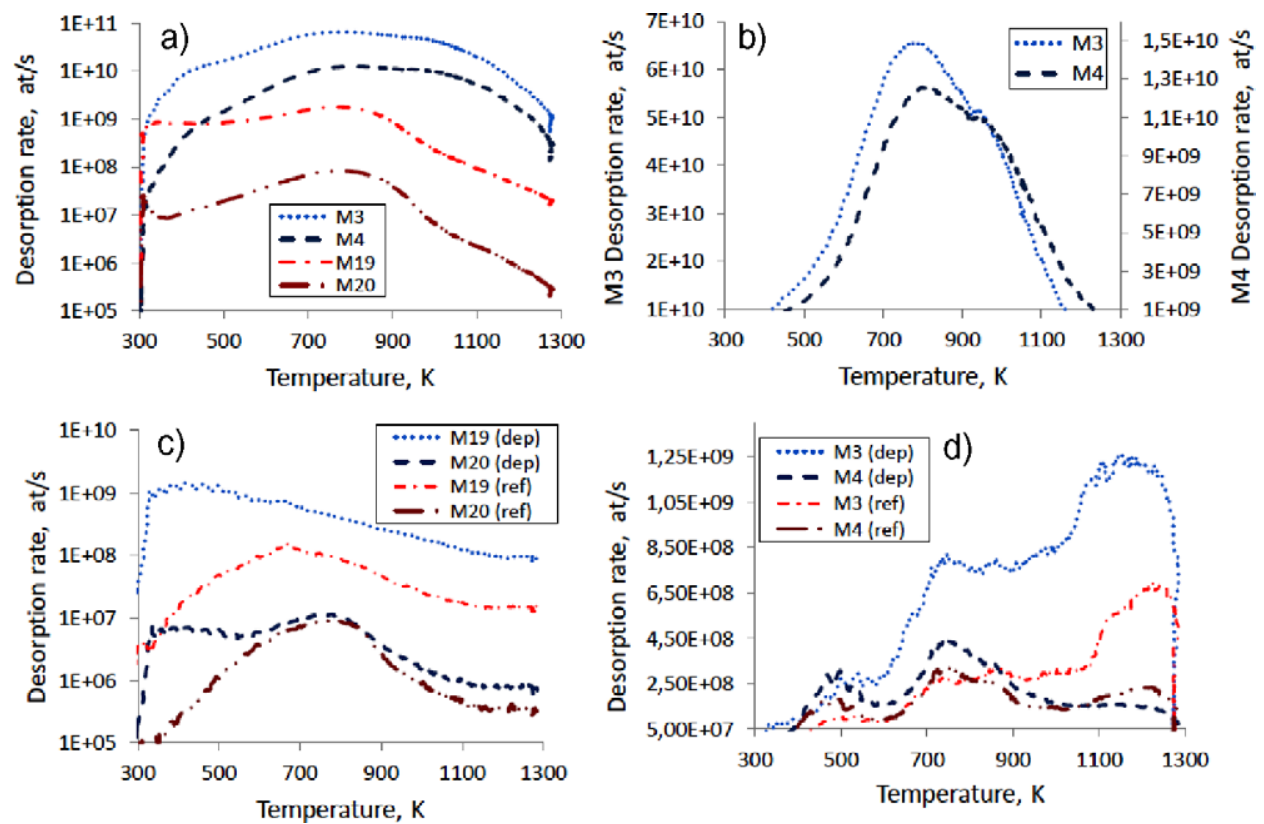

Figure 3. Thermal desorption spectra for the initial and re-exposed deposits on ALT-II and a reference graphite plate. (a) Outgassing curves for M3, M4, M19 and M20 from ALT-II plotted on the logarithmic scale; (b) M3 and M4 spectra from ALT-II plotted in the linear scale to emphasize the peak release around $750 \mathrm{~K}$; (c) release of lower hydrocarbons (M19 and M20) from the re-exposed deposits and the reference pure graphite; (d) release of M3 and M4 from the re-exposed deposits and the reference pure graphite with peaks around 500, 750 and $1150 \mathrm{~K}$.

in figure 4(a). As mentioned earlier, the outgassed deposits are brittle and can be easily detached. Shaping and cutting of the outgassed specimen in order to mount it on the test limiter as well as the exposure to plasma resulted in a loss of some deposits. Images of the re-exposed ALT-II sample and the pure graphite holder are shown in figures 4(b) and (c), respectively. Arrows indicate the direction of the analysis with a ${ }^{3} \mathrm{He}^{+}$beam

The growth of a new deposited layer was observed on the reference graphite plate. The concentration of D atoms along the plate drops exponentially with a characteristic e-folding length $\lambda=0.9-1.0 \mathrm{~cm}$. This value is in agreement with earlier measurements using surface collectors $[19,20]$ and electrical probes [21]. The retention profile on the re-exposed fragment of ALT-II has a plateau region that matches the surface structure and corresponds to the area with the remaining co-deposited layer. Fuel retention in the re-exposed deposit is $1.2 \times 10^{17} \mathrm{D} \mathrm{cm}^{-2}$. This quantity is significantly smaller than the retention in the reference graphite placed at the same radial distance from the plasma: $3.3-1.5 \times 10^{17} \mathrm{D} \mathrm{cm}^{-2}$. The areas where deposits peeled off (positions 1 and $3 \mathrm{~mm}$ in figure 4(a)) also show a lower level of fuel retention in comparison to pure graphite. In the area where the transition between the deposition and erosion zones of the original ALT-II tile starts, the concentrations of D atoms in the studied sample and the reference plate become almost the same. A similar flat profile and lower absolute values of fuel retention were observed in the second experiment in TEXTOR. Depth profiles of the D atoms show a broader distribution of fuel species in the flaking porous deposits than in the pure graphite, thus confirming the results obtained after exposures in PADOS.

Thermal desorption spectra for the re-exposed plate follow the same trend as that measured for the original limiter tile: a single broad desorption peak (650-1000 K) with the maximal desorption rate for M3 and M4 occurring around

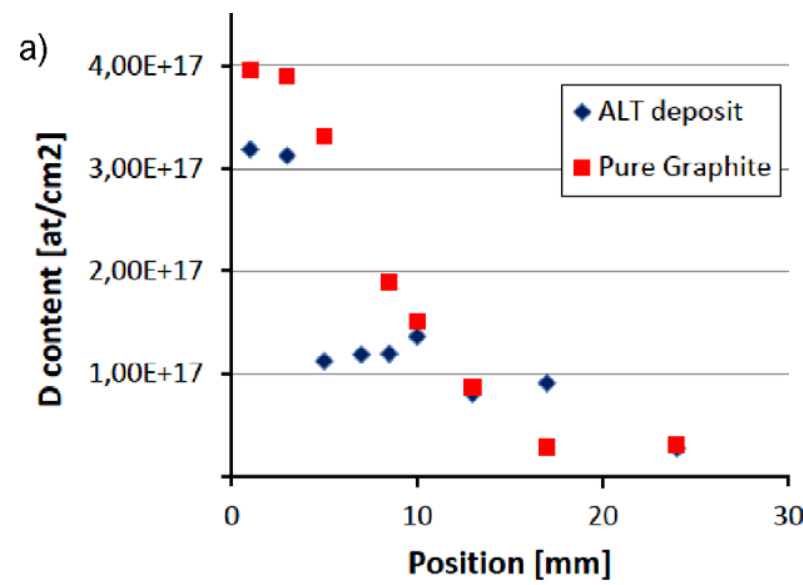

b)

c)
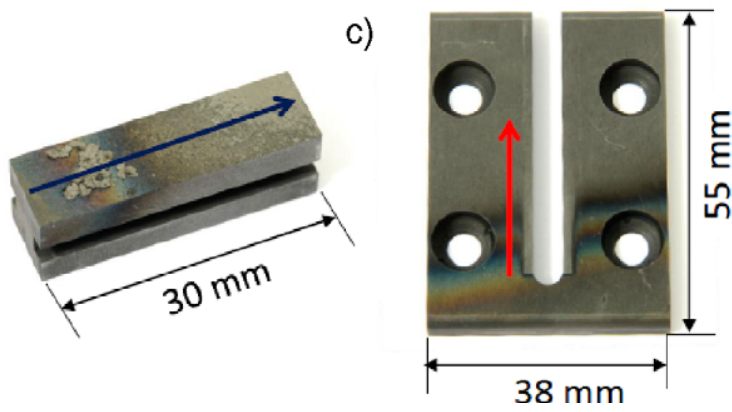

Figure 4. (a) Deuterium content in the re-exposed deposit and reference plates after $40 \mathrm{~s}$ of exposure to SOL plasma in the TEXTOR tokamak; (b) photograph of the re-exposed specimen with the remaining deposit; (c) the pure graphite holder after exposure. Arrows indicate the direction of the NBA scan.

$750 \mathrm{~K}$. However, the desorbed amount of HD and $\mathrm{D}_{2}$ is $30-40$ times smaller than that from the original sample. This proves that there is overall agreement between the ion beam and thermal desorption analysis data for the re-exposed plate. 
For masses M19 and M20 two peaks are observed. The desorption at low temperatures $(<600 \mathrm{~K})$ is due to the presence of deuterated water.

\section{Concluding remarks}

The behaviour of fuel-depleted tokamak co-deposits was studied under repeated exposures to deuterium in a laboratory plasma device and in the TEXTOR tokamak. In both cases, deuterium re-adsorption occurred but the quantity of fuel species was over one order of magnitude smaller than the amount determined by ion beams and TDS in the original layers. This may indicate that PFC surfaces that had been treated by thermal fuel removal methods are not immediately re-saturated by fuel during repeated exposure to tokamak plasma. It should be noted that the thermally treated layers are brittle and easy to peel-off. This leads to dust production, but the fuel content in such dust would be small in comparison to dust generated by laser ablation of co-deposits [22]. As a consequence, it would not create major safety problems related to fuel inventory. To our knowledge, the present paper is the first report of systematic studies on re-adsorption. These early results allow for some optimism in the area of fuel retention and removal. The work is being continued to determine re-adsorption and desorption characteristics following various fuel removal schemes.

\section{Acknowledgments}

This work, supported partly by the European Communities under the Contracts of Association between EURATOM-VR and EURATOM-FZJ, was carried out within the framework of the European Fusion Development Agreement. The views and opinions expressed herein do not necessarily reflect those of the European Commission. This work was partly funded by the Swedish Research Council (VR) through contract no. 621-2009-4138.

Published under license from EURATOM.

\section{References}

[1] Andrew P et al 1999 Fusion Eng. Des. 47243

[2] Coad J P et al 2001 J. Nucl. Mater. 290-293 224

[3] Rubel M et al 2003 J. Nucl. Mater. 313-316 321

[4] Roth J. et al 2008 Plasma Phys. Control. Fusion 50103001

[5] Davis J W et al 1986 J. Nucl. Mater. 138 227-34

[6] Skinner C H et al 2003 J. Nucl. Mater. 313-316 496

[7] Widdowson A et al 2007 J. Nucl. Mater. 365-367 341

[8] Counsell G C et al 2006 Plasma Phys. Control. Fusion 48 B 189

[9] Gasior P et al 2009 J. Nucl. Mater. 390/391 585-8

[10] Schweer B et al 2007 J. Nucl. Mater. 363-365 1375

[11] Douai D et al 2011 J. Nucl. Mater. 415 S1021-S1028

[12] Causey R A et al $1990 \mathrm{~J}$. Nucl. Mater. 176/177 987-91

[13] Rubel M et al 2001 J. Nucl. Mater. 290-293 473

[14] Rubel M et al 2006 Fusion Eng. Des. 81211

[15] Winter J et al 1987 Nucl. Instrum. Methods B 23 538-43

[16] Schweer B et al 2005 Fusion Sci. Technol. 47138

[17] Rubel M et al 2001 Nucl. Fusion 411087

[18] Ivanova D et al 2009 Phys. Scr. T138 014025

[19] Rubel M et al 1995 J. Nucl. Mater. 220-222 536

[20] Emmoth B et al 2003 J. Nucl. Mater. 313-316 729

[21] Lehnen M et al 2003 Nucl. Fusion 43168

[22] Ivanova D et al 2011 J. Nucl. Mater. 415 S801-S804 